\title{
Homogenization approach to the dispersion theory for reactive transport through porous media*
}

\author{
Grégoire Allaire \\ CMAP, Ecole Polytechnique, \\ F-91128 Palaiseau, France \\ (gregoire.allaire@polytechnique.fr) \\ Andro Mikelić . \\ Université de Lyon, Lyon, F-69003, France; \\ Université Lyon 1, Institut Camille Jordan, UMR 5208, \\ 43, Bd du 11 novembre 1918, 69622 Villeurbanne Cedex, France \\ (mikelic@univ-lyon1.fr) \\ Andrey Piatnitski \\ Narvik University College, Postbox 385, Narvik 8505, Norway \\ and Lebedev Physical Institute, Leninski prospect 53, \\ 119991, Moscow, Russia \\ (andrey@sci.lebedev.ru)
}

November 9, 2009

\begin{abstract}
We study the homogenization problem for a convection-diffusion equation in a periodic porous medium in the presence of chemical reaction on the pores surface. Mathematically this model is described in terms of a solution to a system of convection-diffusion equation in the medium and ordinary differential equation defined on the pores surface. These equations are coupled through the boundary condition for the convection-diffusion problem.
\end{abstract}

*The research of G.A. and A.M. was partially supported by the GNR MOMAS CNRS-2439 (Modélisation Mathématique et Simulations numériques liées aux problèmes de gestion des déchets nucléaires) (PACEN/CNRS, ANDRA, BRGM, CEA, EDF, IRSN). G. A. is a member of the DEFI project at INRIA Saclay Ile-de-France and is partially supported by the Chair "Mathematical modelling and numerical simulation, F-EADS - Ecole Polytechnique - INRIA". 
Under an appropriate choice of scaling factors (large Péclet and Damkohler numbers), we obtain the homogenized problem in a moving frame whose effective velocity does actually depend on the chemical reaction.

\section{Introduction}

We consider saturated flow through a porous medium. The flow domain contains a certain mass of solute, usually called tracer. Experimental works show that the tracer gradually spreads with flow, but its spreading is not well described by the simply averaged advection-diffusion equations for the concentration. This spreading phenomenon is called hydrodynamic dispersion.

Following [10], the hydrodynamic dispersion is the averaged macroscopic picture of the motion of the tracer particles through the pore structure and of the chemical reactions of the solute with the solid walls and with other particles. It is caused by two basic transport phenomena involved: convection and molecular diffusion. Their simultaneous presence in the pore structure leads to a complex spreading of the tracer. The interaction between the solid pore interfaces and the fluid is related to the adsorption or deposition of tracer particles on the solid surface. Eventually, radioactive decay and chemical reactions within the fluid may also cause concentration changes.

Due to the complexity of the problem, many results in the literature are concerned with simple models of porous media being either bundles of capillary tubes, or arrays of cells and so on. Such simplifications allow explicit calculations. Taylor's dispersion is one of the most well-known examples of the role of transport in dispersing a flow carrying a dissolved solute. The simplest setting for observing it, is the injection of a solute into a slit channel. The solute is transported by Poiseuille's flow. In this situation Taylor found in [36] an explicit expression for the dispersion.

Actually the hydrodynamic dispersion could be studied in three distinct regimes: a) diffusion-dominated mixing, b) Taylor dispersion-mediated mixing and c) chaotic advection. In the first regime, the velocity is small and the Péclet's number $\mathbf{P e}$ is of order one or smaller. Molecular diffusion plays the dominant role in solute dispersion. This case is well-understood even for reactive flows (see e.g. the papers [16], [18], [20], [21], [22], [17]). If the flow rate is increased so that the Péclet's number $\mathbf{P e}$ is much larger than one, then there is a time scale at which transversal molecular diffusion smears the contact discontinuity into a plug. This is the regime under study in the present paper. In addition to dominant Péclet's number we also consider dominant non-dimensional numbers linked to the chemistry, like Damkohler's number. Eventually the third regime, corresponding to turbulent mixing, is much more delicate and is not considered here.

Our main contribution (see Theorem 3 ) is to give a rigorous derivation of a macroscopic homogenized model explaining Taylor dispersion for a tracer in an incompressible saturated flow through a periodic porous medium, undergoing linear adsorption/desorption chemical reactions on the solid boundaries of the 
pores. Our main technical tool is the notion of two-scale convergence with drift introduced in [23] and applied to convection-diffusion problems in [8] and [14]. With respect to these two previous works the new feature in the present work is the coupling of a convection-diffusion for the bulk solute with an ordinary differential equation for surface concentration.

For the derivation of Taylor's dispersion in porous media using formal two-scale expansions, we refer to [9], [24], [35] and references therein. Volume averaging approach to the effective dispersion for reactive flows through porous media requires an ad hoc closure hypothesis, as in [29].

Rigorous mathematical justification of Taylor's dispersion in capillary tubes, for classical Taylor's case and for reactive flows, was undertaken in [25] and [12]. In the case of oscillating coefficients (a mesoscopic porous medium), with no chemical reactions, the rigorous study of dispersion for dominant Péclet's number, is in [34] and in [11]. The approach from [11] is based on an expansion around the regular solutions for the underlying linear transport equation. This approach requires compatible data but also gives an error estimate. In this paper we deal with the pore geometry and dominant Péclet's and Damköhler's numbers and we think that the two-scale convergence with drift is the right tool to address problems of such level of difficulty.

The contents of the paper is the following. In Section 2 we describe our model and its scaling in terms of various geometrical and physical quantities. Section 3 is devoted to the precise statement of our result, to some uniform a priori estimates and several definitions of two-scale convergence with drift. Section 4 is devoted to a weak convergence proof of our result based on passing to the limit in the variational formulation of the problem with adequate test functions. Finally Section 5 concludes the proof of our main theorem by showing that the two-scale convergence is actually strong. It relies on a $\Gamma$ convergence type result, namely on the convergence of the associated energy. Let us finish this introduction by referring the less mathematically inclined reader to another paper of us [4] where the rigorous two-scale convergence with drift is replaced by simpler two-scale asymptotic expansions with drift and which features some numerical computations of homogenized dispersion tensors.

\section{Statement of the problem and its non-dimensional form}

We consider diffusive transport of the solute particles transported by a stationary incompressible viscous flow through an idealized infinite porous medium. The flow regime is assumed to be laminar through the fluid part $\Omega_{f}$ of this porous medium, which is supposed to be a network of interconnected channels (in other words, we suppose that $\Omega_{f}$ is a connected domain in $\mathbb{R}^{n}, n \geq 2$; usually in the applications $n=2,3$ ). The flow satisfies a slip (non penetrating) condition on the fluid/solid interfaces and $\Omega_{f}$ is saturated by the fluid. Solute 
particles are participants in a chemical reaction with the solid boundaries of the pores. For simplicity we suppose that they do not interact between them.

Reactive transport of a single solute is described by the following model for the solute concentration $c^{*}$ :

$$
\frac{\partial c^{*}}{\partial t^{*}}+\mathbf{v}^{*}\left(x^{*}, t^{*}\right) \cdot \nabla_{x^{*}} c^{*}-D^{*} \Delta_{x^{*}} c^{*}=0 \text { in } \Omega_{f} \times\left(0, T^{*}\right),
$$

where $\mathbf{v}^{*}$ is the fluid velocity, and $D^{*}$ the molecular diffusion (a positive constant). At the solid/fluid boundary $\partial \Omega_{f}$ takes place an assumed linear adsorption process, described by the following equations:

$$
-D^{*} \nabla_{x^{*}} c^{*} \cdot \mathbf{n}=\frac{\partial \hat{c}^{*}}{\partial t^{*}}=\hat{k}^{*}\left(c^{*}-\frac{\hat{c}^{*}}{K^{*}}\right) \quad \text { on } \partial \Omega_{f} \times\left(0, T^{*}\right),
$$

where $\hat{k}^{*}$ represents the rate constant for adsorption, $K^{*}$ the linear adsorption equilibrium constant and $\mathbf{n}$ is the unit normal at $\partial \Omega_{f}$ oriented outwards with respect to $\Omega_{f}$. For more on mathematical modeling of adsorption/desorption and references from the chemical engineering we refer to [15].

This system is generic and appears in numerous situations (see e.g. the reference books [19], [30], or [32]). In the modeling variant [4] of this paper, oriented to the chemical engineering readership, we explain in detail how to reduce the linearized models for binary ion exchange, and linearized reactive flow systems with $m$ species to the system (1)-(2).

To make an asymptotic analysis of this problem we must first introduce appropriate scales deduced from characteristic parameters such as the characteristic concentration $c_{R}$, the characteristic length $L_{R}$, the characteristic velocity $V_{R}$, the characteristic diffusivity $D_{R}$, the characteristic time $T_{R}$, and other characteristic quantities denoted by a $R$-index (meaning "reference"). Scaling in homogenization is an important issue (see e.g. [31], [33]). The characteristic length $L_{R}$ coincides in fact with the "observation distance". We assume that the typical heterogeneities in $\Omega_{f}$ have a characteristic size $\ell<<L_{R}$. We set $\varepsilon=\frac{\ell}{L_{R}}<<1$ and the rescaled flow domain is now $\Omega_{\varepsilon}=\Omega_{f} / L_{R}$, with notation reminding us that it contains pores of characteristic non-dimensional size $\varepsilon$. Setting

$$
\begin{aligned}
& u_{f}=\frac{c^{*}}{c_{R}}, x=\frac{x^{*}}{L_{R}}, t=\frac{t^{*}}{T_{R}}, \mathbf{v}(x, t)=\frac{1}{V_{R}} \mathbf{v}^{*}\left(x^{*}, t^{*}\right), D=\frac{D^{*}}{D_{R}}, \\
& k=\frac{\hat{k}^{*}}{k_{R}}, v_{s}=\frac{\hat{c}^{*}}{\hat{c}_{R}}, K=\frac{K^{*}}{K_{R}},
\end{aligned}
$$

we obtain the dimensionless equations

$$
\frac{\partial u_{f}}{\partial t}+\frac{V_{R} T_{R}}{L_{R}} \mathbf{v}(x, t) \cdot \nabla_{x} u_{f}-\frac{D_{R} T_{R}}{L_{R}^{2}} D \Delta_{x} u_{f}=0 \quad \text { in } \Omega_{\varepsilon} \times(0, T)
$$

and

$$
-\frac{D D_{R}}{L_{R}} c_{R} \nabla_{x} u_{f} \cdot \mathbf{n}=\frac{\hat{c}_{R}}{T_{R}} \frac{\partial v_{s}}{\partial t}=k_{R} k\left(c_{R} u_{f}-\frac{\hat{c}_{R} v_{s}}{K K_{R}}\right) \quad \text { on } \partial \Omega_{\varepsilon} \times(0, T) .
$$

This problem involves the following time scales: 
$T_{L}=$ characteristic global advection time scale $=L_{R} / V_{R}$

$T_{D}=$ characteristic global diffusion time scale $=L_{R}^{2} / D_{R}$

$T_{D E}=K_{R} / k_{R}$ (characteristic desorption time)

$T_{A}=\hat{c}_{R} /\left(c_{R} k_{R}\right)$ (characteristic adsorption time)

$T_{\text {react }}=$ superficial chemical reaction time scale $=L_{R} / k_{R}$

and the following characteristic non-dimensional numbers

$\mathbf{P e}=\frac{L_{R} V_{R}}{D_{R}}=\frac{T_{D}}{T_{L}}$ (Péclet number);

$\mathbf{D a}=\frac{L_{R} k_{R}}{D_{R}}=\frac{T_{D}}{T_{\text {react }}}$ (Damkohler number $)$

We choose to study a regime for which $T_{R}=T_{D}$. Due to the complex geometry and in presence of dominant Péclet and Damkohler numbers, solving the full problem for arbitrary values of coefficients is costly and practically impossible. Consequently, one would like to find the effective (or averaged or homogenized) values of the dispersion coefficient and the transport velocity and an effective corresponding parabolic equation for the effective concentration, valid in an infinite homogeneous porous media.

Let us be a little more precise on the definition of $\Omega_{\varepsilon}$. From now on we assume that $\Omega_{\varepsilon}$ is an $\varepsilon$-periodic unbounded open subset of $\mathbb{R}^{n}$. It is built from $\mathbb{R}^{n}$ by removing a periodic distributions of solid obstacles which, after rescaling, are all similar to the unit obstacle $\Sigma^{0}$. More precisely, the unit periodicity cell is identified with the flat unit torus $\mathbb{T}^{n}$ on which we consider a smooth partition $\Sigma^{0} \cup Y^{0}$ where $\Sigma^{0}$ is the solid part and $Y^{0}$ is the fluid part. The fluid part is assumed to be a smooth connected open subset (no assumption is made on the solid part). We define $Y_{\varepsilon}^{j}=\varepsilon\left(Y^{0}+j\right), \Sigma_{\varepsilon}^{j}=$ $\varepsilon\left(\Sigma^{0}+j\right), S_{\varepsilon}^{j}=\varepsilon\left(\partial \Sigma^{0}+j\right), \Omega_{\varepsilon}=\bigcup_{j \in \mathbb{Z}^{n}} Y_{\varepsilon}^{j}$ and $S_{\varepsilon}=\partial \Omega_{\varepsilon}$.

The equations for $u_{\varepsilon}=u_{f}$ and $v_{\varepsilon}=v_{s}$ in their non-dimensional form read (with the velocity $\mathbf{v}_{\varepsilon}=\mathbf{v}$ )

$$
\begin{gathered}
\frac{\partial u_{\varepsilon}}{\partial t}+\operatorname{Pe}_{\varepsilon}(x, t) \cdot \nabla_{x} u_{\varepsilon}=D \Delta_{x} u_{\varepsilon} \quad \text { in } \Omega_{\varepsilon} \times(0, T) \\
u_{\varepsilon}(x, 0)=u^{0}(x), \quad x \in \Omega_{\varepsilon}, \\
-D \nabla_{x} u_{\varepsilon} \cdot \mathbf{n}=\frac{T_{A}}{T_{\text {react }}} \frac{\partial v_{\varepsilon}}{\partial t}=\frac{T_{D}}{T_{\text {react }}} k\left(u_{\varepsilon}-\frac{T_{A}}{T_{D E}} \frac{v_{\varepsilon}}{K}\right) \text { on } \partial \Omega_{\varepsilon} \times(0, T) \\
v_{\varepsilon}(x, 0)=v^{0}(x), \quad x \in \partial \Omega_{\varepsilon} .
\end{gathered}
$$

In Section 3.1 we shall make some further assumptions on the scaling of the above adimensional system in terms of the geometrical small parameter $\varepsilon$.

\section{Main results}

\subsection{Assumptions and main convergence theorem}

In the present work we make the following two hypothesis. 
(H1)

$$
\mathbf{P e}=\frac{1}{\varepsilon}, \quad \mathbf{D a}=\frac{T_{D}}{T_{\text {react }}}=\frac{1}{\varepsilon}, \quad \frac{T_{A}}{T_{\text {react }}}=\varepsilon, \quad \frac{T_{A}}{T_{D E}}=1 .
$$

(H2) The velocity field is periodic and incompressible, i.e.

$$
\mathbf{v}_{\varepsilon}(x, t)=\mathbf{b}\left(\frac{x}{\varepsilon}\right)
$$

with a periodic divergence-free vector field $\mathbf{b}(y)$ satisfying

$$
|\mathbf{b}(y)| \in L^{\infty}\left(\mathbb{R}^{n}\right), \quad \operatorname{div}_{y} \mathbf{b}(y)=0 \quad \text { in } Y^{0}, \quad \mathbf{b}(y) \cdot \mathbf{n}(y)=0 \quad \text { on } \partial \Sigma^{0} .
$$

The initial data are chosen such that $u^{0}(x) \in L^{2}\left(\mathbb{R}^{n}\right)$ and $v^{0}(x) \in H^{1}\left(\mathbb{R}^{n}\right)$. Taking into account (9), we rewrite problem (5)-(8) as follows

$$
\begin{gathered}
\partial_{t} u_{\varepsilon}+\frac{1}{\varepsilon} \mathbf{b}\left(\frac{x}{\varepsilon}\right) \cdot \nabla u_{\varepsilon}-D \Delta u_{\varepsilon}=0 \quad \text { in } \Omega_{\varepsilon} \times(0, T), \\
-\frac{D}{\varepsilon} \frac{\partial u_{\varepsilon}}{\partial \mathbf{n}}=\partial_{t} v_{\varepsilon}=\frac{k}{\varepsilon^{2}}\left(u_{\varepsilon}-\frac{v_{\varepsilon}}{K}\right) \quad \text { on } \partial \Omega_{\varepsilon} \times(0, T), \\
u_{\varepsilon}(x, 0)=u^{0}(x), \quad v_{\varepsilon}(x, 0)=v^{0}(x),
\end{gathered}
$$

where we recall that $K$ and $k$ are positive constants. The variational formulation of (10)-(11) is: find $u_{\varepsilon}(t, x) \in L^{2}\left((0, T) ; H^{1}\left(\Omega_{\varepsilon}\right)\right) \cap C^{0}\left([0, T] ; L^{2}\left(\Omega_{\varepsilon}\right)\right)$ and $v_{\varepsilon}(t, x) \in C^{0}\left([0, T] ; L^{2}\left(\partial \Omega_{\varepsilon}\right)\right)$ such that, for any test functions $\phi(x) \in H^{1}\left(\Omega_{\varepsilon}\right)$, $\psi(x) \in L^{2}\left(\partial \Omega_{\varepsilon}\right)$, and a.e. in time,

$$
\begin{gathered}
\frac{d}{d t} \int_{\Omega_{\varepsilon}} u_{\varepsilon} \phi+\frac{1}{\varepsilon} \int_{\Omega_{\varepsilon}} \mathbf{b}\left(\frac{x}{\varepsilon}\right) \cdot \nabla u_{\varepsilon} \phi+\int_{\Omega_{\varepsilon}} D \nabla u_{\varepsilon} \cdot \nabla \phi+\frac{k}{\varepsilon} \int_{\partial \Omega_{\varepsilon}}\left(u_{\varepsilon}-\frac{v_{\varepsilon}}{K}\right) \phi=0, \\
\frac{d}{d t} \int_{\partial \Omega_{\varepsilon}} v_{\varepsilon} \psi-\frac{k}{\varepsilon^{2}} \int_{\partial \Omega_{\varepsilon}}\left(u_{\varepsilon}-\frac{v_{\varepsilon}}{K}\right) \psi=0
\end{gathered}
$$

together with the initial condition (12).

Remark 1. If the velocity field $\mathbf{b}(y)$ is not divergence-free and/or does not satisfy the no-penetration condition $\mathbf{b}(y) \cdot \mathbf{n}(y)=0$ on $\partial \Sigma^{0}$, it is still possible to homogenize (10)-(12) by using first a factorization principle in the spirit of $[8]$.

Remark 2. We do not know how to extend our analysis to the case of a macroscopically modulated velocity field $\mathbf{b}(x, y)$. Actually we believe the asymptotic behavior could be completely different, according to the precise assumptions on $\mathbf{b}(x, y)$. For example, in [6] (for a convection-diffusion equation) and [7] (for a self-adjoint diffusion equation) it was shown, under specific geometric assumptions on the macroscopic dependence of the coefficients, that a localization effect can take place at a lengthscale of $\sqrt{\epsilon}$. However the general case is still open and it is very likely that localization does not always happen. 
To simplify the presentation we use an extension operator from the perforated domain $\Omega_{\varepsilon}$ into $\mathbb{R}^{n}$ (although it is not necessary). As was proved in [1], there exists such an extension operator $T^{\varepsilon}$ from $H^{1}\left(\Omega_{\varepsilon}\right)$ in $H^{1}\left(\mathbb{R}^{n}\right)$ satisfying $\left.T^{\varepsilon} \psi\right|_{\Omega_{\varepsilon}}=\psi$ and the inequalities

$$
\|\psi\|_{L^{2}\left(\mathbb{R}^{n}\right)} \leq C\|\psi\|_{L^{2}\left(\Omega_{\varepsilon}\right)}, \quad\|\nabla \psi\|_{L^{2}\left(\mathbb{R}^{n}\right)} \leq C\|\nabla \psi\|_{L^{2}\left(\Omega_{\varepsilon}\right)}
$$

with a constant $C$ independent of $\varepsilon$, for any $\psi \in H^{1}\left(\Omega_{\varepsilon}\right)$. We keep for the extended function $T^{\varepsilon} \psi_{\varepsilon}$ the same notation $\psi_{\varepsilon}$. Our main result is the following strong convergence.

Theorem 3. The sequence $\left\{u_{\varepsilon}, v_{\varepsilon}\right\}$ of solutions to (10)-(12) satisfies

$$
u_{\varepsilon}(t, x)=u\left(t, x-\frac{\overline{\mathbf{b}}}{\varepsilon} t\right)+r_{\varepsilon}^{u}(t, x), v_{\varepsilon}(t, x)=K u\left(t, x-\frac{\overline{\mathbf{b}}}{\varepsilon} t\right)+r_{\varepsilon}^{v}(t, x)
$$

with

$$
\lim _{\varepsilon \rightarrow 0} \int_{0}^{T} \int_{\mathbb{R}^{n}}\left|r_{\varepsilon}^{u}(t, x)\right|^{2} d t d x=0 \quad \text { and } \quad \lim _{\varepsilon \rightarrow 0} \varepsilon \int_{0}^{T} \int_{\partial \Omega_{\varepsilon}}\left|r_{\varepsilon}^{v}(t, x)\right|^{2} d t d x=0
$$

where $\overline{\mathbf{b}}$ is the so-called effective drift (a constant vector) given by

$$
\overline{\mathbf{b}}=\left(\left|Y^{0}\right|+\left|\partial \Sigma^{0}\right|_{n-1} K\right)^{-1} \int_{Y^{0}} \mathbf{b}(y) d y
$$

and $u(x, t)$ is the unique solution of the homogenized problem

$$
\left\{\begin{array}{l}
\left(\left|Y^{0}\right|+K\left|\partial \Sigma^{0}\right|_{n-1}\right) \partial_{t} u=\operatorname{div}_{x}\left(A^{*} \nabla_{x} u\right) \text { in } \mathbb{R}^{n} \times(0, T), \\
u(x, 0)=\frac{\left|Y^{0}\right| u^{0}(x)+\left|\partial \Sigma^{0}\right|_{n-1} v^{0}(x)}{\left|Y^{0}\right|+K\left|\partial \Sigma^{0}\right|_{n-1}} \text { in } \mathbb{R}^{n},
\end{array}\right.
$$

where the effective diffusion tensor $A^{*}$ is defined by

$$
A^{*}=\frac{K^{2}}{k}\left|\partial \Sigma^{0}\right|_{n-1} \overline{\mathbf{b}} \otimes \overline{\mathbf{b}}+D \int_{Y^{0}}\left(\mathbf{I}+\nabla_{y} \chi(y)\right)\left(\mathbf{I}+\nabla_{y} \chi(y)\right)^{\mathcal{T}} d y .
$$

The vector-valued periodic function $\chi$ has components $\chi_{i} \in H^{1}\left(Y^{0}\right)$ which are solutions of the following cell problem, $1 \leq i \leq n$,

$$
\begin{gathered}
\mathbf{b}(y) \cdot \nabla \chi_{i}(y)-D \operatorname{div}\left(\nabla\left(\chi_{i}(y)+y_{i}\right)\right)=\bar{b}_{i}-b_{i}(y) \quad \text { in } Y^{0}, \\
D \nabla\left(\chi_{i}(y)+y_{i}\right) \cdot \mathbf{n}=K \bar{b}_{i} \quad \text { on } \partial \Sigma^{0} .
\end{gathered}
$$

Here $\left|Y^{0}\right|$ stands for the volume of $Y^{0},\left|\partial \Sigma^{0}\right|_{n-1}$ for the $(n-1)$-dimensional measure of the boundary $\partial \Sigma^{0}$ and $\mathbf{n}(y)$ is the external unit normal on $\partial \Sigma^{0}$.

Remark 4. Convection is not seen in the homogenized equation (14) because the solution $u$ is defined in moving coordinates when compared to $u_{\varepsilon}$ and $v_{\varepsilon}$ in (13). However, (14) is equivalent to a convection diffusion equation by a 
simple change of reference frame. Indeed, introducing $\tilde{u}_{\varepsilon}(t, x)=u\left(t, x-\frac{\overline{\mathbf{b}}}{\varepsilon} t\right)$, it is a solution of

$$
\begin{cases}\frac{\partial \tilde{u}_{\varepsilon}}{\partial t}+\frac{1}{\varepsilon} \overline{\mathbf{b}} \cdot \nabla \tilde{u}_{\varepsilon}-\operatorname{div}\left(A^{*} \nabla \tilde{u}_{\varepsilon}\right)=0 & \text { in } \mathbb{R}^{n} \times(0, T) \\ \tilde{u}_{\varepsilon}(t=0, x)=\frac{\left|Y^{*}\right| u^{0}(x)+|\partial \mathcal{O}|_{n-1} v^{0}(x)}{\left|Y^{*}\right|+K|\partial \mathcal{O}|_{n-1}} & \text { in } \mathbb{R}^{n}\end{cases}
$$

Theorem 3 is valid only for an unbounded domain $\Omega_{\varepsilon}$. It is clear from the large drift in (13) or in the above equation that there is a serious difficulty to deal with the case of a bounded domain for time much larger than $\varepsilon$.

Remark 5. The adsorption rate $k$ appears only in the first term of the right hand side of (15), so it is easy to check that $A^{*}$ varies monotonically with $k$ and blows up when $k$ goes to 0 . Since in the original problem (10)-(12) the limit case $k=0$ is perfectly legitimate and means no chemical reaction at all, this shows that the homogenization limit does not commute with the limit as $k$ goes to 0 . When $k$ goes to $+\infty$, the first term of the right hand side of (15) cancels out, a situation which corresponds to fixing $v_{\varepsilon}=K u_{\varepsilon}$ on the pore boundaries. The dependence of $A^{*}$ upon the equilibrium constant $K$ is implicit. At least formally, when $K$ goes to 0 , one recover the usual cell problem, drift and homogenized tensor corresponding to homogeneous Neumann boundary condition on the pore boundaries (i.e. without chemistry). On the other hand when $K$ goes to $+\infty$, we obtain that $\overline{\mathbf{b}}=0$ and the product $K \overline{\mathbf{b}}$, as well as $A^{*}$, have a non-zero limit, corresponding to the case of Fourier or Robin boundary condition for $u_{\varepsilon}$.

Remark 6. The proof of Theorem 3 is the focus of the last two sections and relies on the notion of two-scale convergence with drift. For the mathematically less inclined reader, a formal method for guessing the correct homogenized problem (14) is the method of two-scale asymptotic expansions with drift (see [4] and [28]). More precisely, one assumes that

$$
u_{\varepsilon}(t, x)=\sum_{i=0}^{+\infty} \varepsilon^{i} u_{i}\left(t, x-\frac{\overline{\mathbf{b}}}{\varepsilon} t, \frac{x}{\varepsilon}\right),
$$

with $u_{i}(t, x, y)$ a function of the macroscopic variable $x$ and of the periodic microscopic variable $y \in Y=(0,1)^{n}$, and similarly

$$
v_{\varepsilon}(t, x)=\sum_{i=0}^{+\infty} \varepsilon^{i} v_{i}\left(t, x-\frac{\overline{\mathbf{b}}}{\varepsilon} t, \frac{x}{\varepsilon}\right)
$$

Plugging these ansatz in the equation (10) yields after some standard algebra the desired result, at least formally.

\subsection{Uniform a priori estimates}

We now derive a priori estimates based on the energy equality. As usual, they imply existence of a unique solution to problem (10)-(12). Depending 
on the assumed regularity of the initial data, we could prove arbitrary high regularity of the solution.

Lemma 1. There exists a constant $C$, which does not depend on $\varepsilon$, such that the solution of (10)-(12) satisfies

$$
\begin{gathered}
\left\|u_{\varepsilon}\right\|_{L^{\infty}\left((0, T) ; L^{2}\left(\Omega_{\varepsilon}\right)\right)}+\sqrt{\varepsilon}\left\|v_{\varepsilon}\right\|_{L^{\infty}\left((0, T) ; L^{2}\left(S_{\varepsilon}\right)\right)}+\left\|\nabla u_{\varepsilon}\right\|_{L^{2}\left((0, T) \times \Omega_{\varepsilon}\right)} \\
\leq C\left(\left\|u^{0}\right\|_{L^{2}\left(\mathbb{R}^{n}\right)}+\left\|v^{0}\right\|_{H^{1}\left(\mathbb{R}^{n}\right)}\right) .
\end{gathered}
$$

Proof. The energy estimate for (10)-(12) reads

$$
\begin{gathered}
\frac{1}{2} \frac{d}{d t}\left[\left\|u_{\varepsilon}\right\|_{L^{2}\left(\Omega_{\varepsilon}\right)}^{2}+\frac{\varepsilon}{K}\left\|v_{\varepsilon}\right\|_{L^{2}\left(\partial \Omega_{\varepsilon}\right)}^{2}\right]+ \\
\int_{\Omega_{\varepsilon}} D \nabla u_{\varepsilon}(t) \cdot \nabla u_{\varepsilon}(t) d x+\frac{\varepsilon k}{\varepsilon^{2}} \int_{S_{\varepsilon}}\left(u_{\varepsilon}-\frac{v_{\varepsilon}}{K}\right)^{2} d \sigma=0
\end{gathered}
$$

from which we easily deduce the desired result since $\varepsilon\left\|v^{0}\right\|_{L^{2}\left(\partial \Omega_{\varepsilon}\right)}^{2} \leq C\left\|v^{0}\right\|_{H^{1}\left(\mathbb{R}^{n}\right)}^{2}$. To obtain (18) we multiply equation (10) by $u_{\varepsilon}$ and integrate by parts over $\Omega_{\varepsilon}$. The convective term cancels out since the velocity is divergence-free and has a zero normal component on the boundary

$$
\int_{\Omega_{\varepsilon}} \mathbf{b}\left(\frac{x}{\varepsilon}\right) \cdot \nabla u_{\varepsilon} u_{\varepsilon} d x=\frac{1}{2} \int_{\Omega_{\varepsilon}} \operatorname{div}\left(\mathbf{b}\left(\frac{x}{\varepsilon}\right)\left|u_{\varepsilon}\right|^{2}\right) d x=-\frac{1}{2} \int_{S_{\varepsilon}}\left|u_{\varepsilon}\right|^{2} \mathbf{b}\left(\frac{x}{\varepsilon}\right) \cdot \mathbf{n} d \sigma=0 .
$$

It yields

$$
\frac{1}{2} \frac{d}{d t}\left\|u_{\varepsilon}\right\|_{L^{2}\left(\Omega_{\varepsilon}\right)}^{2}+\int_{\Omega_{\varepsilon}} D \nabla u_{\varepsilon}(t) \cdot \nabla u_{\varepsilon}(t) d x+\frac{k}{\varepsilon} \int_{S_{\varepsilon}}\left(u_{\varepsilon}^{2}-\frac{v_{\varepsilon}}{K} u_{\varepsilon}\right) d \sigma=0 .
$$

Multiplying then the equation $\partial_{t} v_{\varepsilon}=\frac{k}{\varepsilon^{2}}\left(u_{\varepsilon}-\frac{v_{\varepsilon}}{K}\right)$ by $\varepsilon v_{\varepsilon} / K$ and integrating the result over $S_{\varepsilon}$ yields

$$
\frac{1}{2} \frac{\varepsilon}{K} \frac{d}{d t}\left\|v_{\varepsilon}\right\|_{L^{2}\left(S_{\varepsilon}\right)}^{2}+\frac{k}{\varepsilon} \int_{S_{\varepsilon}}\left(-\frac{u_{\varepsilon} v_{\varepsilon}}{K}+\frac{v_{\varepsilon}^{2}}{K^{2}}\right) d \sigma=0 .
$$

Summing up the last two relations, we obtain (18).

Next we estimate $v_{\varepsilon}$ using $u_{\varepsilon}$. Without loss of generality we can assume that the function $v_{\varepsilon}$ is defined by the equation $\partial_{t} v_{\varepsilon}=\frac{k}{\varepsilon^{2}}\left(u_{\varepsilon}-\frac{v_{\varepsilon}}{K}\right)$ everywhere in $\Omega_{\varepsilon}$ and not solely on $S_{\varepsilon}$.

Lemma 2. There exists a constant $C$, which does not depend on $\varepsilon$, such that

$$
\left\|v_{\varepsilon}\right\|_{L^{2}\left((0, T) ; H^{1}\left(\Omega_{\varepsilon}\right)\right)} \leq C\left(\left\|u_{\varepsilon}\right\|_{L^{2}\left((0, T) ; H^{1}\left(\Omega_{\varepsilon}\right)\right)}+\varepsilon\left\|v^{0}\right\|_{H^{1}\left(\mathbb{R}^{n}\right)}\right) .
$$


Proof. Solving explicitly the ODE (11), we get

$$
v_{\varepsilon}(t, x)=\int_{0}^{t} \frac{k}{\varepsilon^{2}} \exp \left(\frac{k}{K \varepsilon^{2}}(s-t)\right) u_{\varepsilon}(s, x) d s+v^{0}(x) \exp \left(-\frac{k t}{K \varepsilon^{2}}\right) .
$$

Since $\frac{k}{\varepsilon^{2}} \exp \left\{-\frac{k \tau}{K \varepsilon^{2}}\right\} \chi_{0 \leq \tau \leq t}$ is bounded in $L^{1}(0, T)$ independently of $\varepsilon$, Young's inequality yields

$$
\left\{\begin{array}{l}
\left\|v_{\varepsilon}\right\|_{L^{2}\left((0, T) ; H^{1}\left(\Omega_{\varepsilon}\right)\right)} \leq C\left(\left\|u_{\varepsilon}\right\|_{L^{2}\left((0, T) ; H^{1}\left(\Omega_{\varepsilon}\right)\right)}+\varepsilon\left\|v^{0}\right\|_{H^{1}\left(\mathbb{R}^{n}\right)}\right) \\
\left\|v_{\varepsilon}\right\|_{L^{2}\left((0, T) \times \Omega_{\varepsilon}\right)} \leq C\left(\left\|u_{\varepsilon}\right\|_{\left.L^{2}\left((0, T) \times \Omega_{\varepsilon}\right)\right)}+\varepsilon\left\|v^{0}\right\|_{L^{2}\left(\mathbb{R}^{n}\right)}\right)
\end{array}\right.
$$

The next a priori estimate is again a consequence of the energy equality (18).

Lemma 3. There exists another constant $C$, which does not depend on $\varepsilon$, such that

$$
\left\|\frac{1}{K} v_{\varepsilon}-u_{\varepsilon}\right\|_{L^{2}\left((0, T) \times \Omega_{\varepsilon}\right)} \leq C \varepsilon .
$$

Proof. The desired estimate is a consequence of the following Poincaré type inequality

$$
\|w\|_{L^{2}\left(\Omega_{\varepsilon}\right)}^{2} \leq C\left(\varepsilon^{2}\|\nabla w\|_{L^{2}\left(\Omega_{\varepsilon}\right)}^{2}+\varepsilon\|w\|_{L^{2}\left(\partial \Omega_{\varepsilon}\right)}^{2}\right)
$$

This inequality is derived in [13]. Combining it with the energy estimate (18), we obtain the statement of lemma.

Remark 7. All the previous a priori estimates are not uniform with respect to $k$ and $K$. This is one reason why taking the homogenization limit $\varepsilon \rightarrow 0$ and taking the zero-adsorption limit $k \rightarrow 0$ do not commute.

\subsection{Two-scale convergence with drift}

For the reader's convenience we recall here the definition of two-scale convergence in moving coordinates (or with drift) introduced in [23] (see [3] for a pedagogical presentation including detailed proofs).

Definition 1. Let $\mathcal{V}$ be a constant vector in $\mathbb{R}^{n}$. We say that a sequence of functions $U_{\varepsilon}(t, x) \in L^{2}\left((0, T) \times \mathbb{R}^{n}\right)$ two-scale converges in moving coordinates (or, equivalently, with drift) $(x, t) \rightarrow\left(x-\frac{\mathcal{V}}{\varepsilon} t, t\right)$ to a function $U_{0}(t, x, y) \in$ $L^{2}\left((0, T) \times \mathbb{R}^{n} \times \mathbb{T}^{n}\right)$ if

$$
\left\|U_{\varepsilon}\right\|_{L^{2}\left((0, T) \times \mathbb{R}^{n}\right)} \leq C
$$

and for any $\phi(t, x, y) \in C_{0}^{\infty}\left((0, T) \times \mathbb{R}^{n} \times \mathbb{T}^{n}\right)$

$$
\lim _{\varepsilon \rightarrow 0} \int_{0}^{T} \int_{\mathbb{R}^{n}} U_{\varepsilon}(t, x) \phi\left(t, x-\frac{\mathcal{V}}{\varepsilon} t, \frac{x}{\varepsilon}\right) d x d t=\int_{0}^{T} \int_{\mathbb{R}^{n}} \int_{\mathbb{T}^{n}} U_{0}(t, x, y) \phi(t, x, y) d x d y d t .
$$

The convergence (21) is denoted by $U_{\varepsilon} \stackrel{2-d r i f t}{\longrightarrow} U_{0}$. 
In analogy with the classic two-scale convergence results (see [2] and [26]), we have:

Proposition 8. ([23]) Let $\mathcal{V}$ be a constant vector in $\mathbb{R}^{n}$ and let the sequence $U_{\varepsilon}$ be uniformly bounded in $L^{2}\left((0, T) ; H^{1}\left(\mathbb{R}^{n}\right)\right)$. Then there exists a subsequence, still denoted by $\varepsilon$, and functions $U_{0}(t, x) \in L^{2}\left((0, T) ; H^{1}\left(\mathbb{R}^{n}\right)\right)$ and $U_{1}(t, x, y) \in L^{2}\left((0, T) \times \mathbb{R}^{n} ; H^{1}\left(\mathbb{T}^{n}\right)\right)$ such that

$$
\begin{gathered}
U_{\varepsilon} \stackrel{2-d r i f t}{\longrightarrow} U_{0}, \\
\nabla U_{\varepsilon} \stackrel{2-d r i f t}{\longrightarrow} \nabla_{x} U_{0}+\nabla_{y} U_{1} .
\end{gathered}
$$

Thanks to estimate (18) and Lemmata 1, 2 and 3 and Proposition 8, we have the following compactness result.

Corollary 9. Let $\left\{u_{\varepsilon}, v_{\varepsilon}\right\}$ be the solution of problem (10)-(12), extended to the whole space. Take the drift $\mathcal{V}=\overline{\mathbf{b}}$. Then there exists a subsequence (still denoted by $\varepsilon)$ and $\{u, w, q\} \in L^{2}\left((0, T) ; H^{1}\left(\mathbb{R}^{n}\right)\right) \times L^{2}\left((0, T) \times \mathbb{R}^{n} ; H^{1}\left(\mathbb{T}^{n}\right)\right) \times$ $L^{2}\left((0, T) \times \mathbb{R}^{n} \times \mathbb{T}^{n}\right)$ such that

$$
\left\{\begin{array}{l}
u_{\varepsilon} \stackrel{2-\text { drift }}{\longrightarrow} u(t, x), \quad \nabla u_{\varepsilon} \stackrel{2-d r i f t}{\longrightarrow} \nabla u(t, x)+\nabla_{y} w(t, x, y), \\
v_{\varepsilon} \stackrel{2-d r i f t}{\longrightarrow} K u(t, x), \quad \frac{1}{\varepsilon}\left(\frac{v_{\varepsilon}}{K}-u_{\varepsilon}\right) \stackrel{2-d r i f t}{\longrightarrow} q(t, x, y) .
\end{array}\right.
$$

The fact that $\left\{u_{\varepsilon}\right\}$ and $\left\{v_{\varepsilon} / K\right\}$ have the same (two-scale with drift) limits follows from Lemma 3 .

Next we show that the last convergence in (24) holds true even for integrals on the boundary $\partial \Omega_{\varepsilon}$ instead of $\Omega_{\varepsilon}$. This result is reminiscent of the notion of two-scale convergence on periodic surfaces developed in [5] and [27] and we could develop the same convergence but with drift.

Corollary 10. Let $\left\{u_{\varepsilon}, v_{\varepsilon}\right\}$ be as in Corollary 9. Then, for the same two-scale limit with drift $q$, as defined in (24), we have

$$
\begin{gathered}
\lim _{\varepsilon \rightarrow 0} \varepsilon \int_{0}^{T} \int_{\partial \Omega_{\varepsilon}} \frac{1}{\varepsilon}\left(u_{\varepsilon}-v_{\varepsilon} / K\right) \phi\left(t, x-\frac{\overline{\mathbf{b}} t}{\varepsilon}, \frac{x}{\varepsilon}\right) d \sigma d t= \\
\int_{0}^{T} \int_{\partial \Sigma^{0}} \int_{\mathbb{R}^{n}} q(t, x, y) \phi(t, x, y) d x d \sigma_{y} d t,
\end{gathered}
$$

for any test function $\phi(t, x, y) \in C_{0}^{\infty}\left((0, T) \times \mathbb{R}^{n} \times \mathbb{T}^{n}\right)$,

Proof. Let $\mathbf{a} \in C^{1}\left(\bar{Y}^{0} ; \mathbb{R}^{n}\right)$ be a solution for

$$
\mathbf{a} \cdot \mathbf{n}=1 \text { on } \partial \Sigma^{0} ; \quad \operatorname{div} \mathbf{a}=\frac{\left|\partial \Sigma^{0}\right|_{n-1}}{\left|Y^{0}\right|} \text { in } Y^{0} ; \mathbf{a} \text { is } Y \text {-periodic, }
$$


and let $\mathbf{a}^{\varepsilon}(x)=\varepsilon \mathbf{a}(x / \varepsilon)$ in $\Omega_{\varepsilon}$. Then we have

$$
\begin{gathered}
\int_{0}^{T} \int_{\partial \Omega_{\varepsilon}}\left(u_{\varepsilon}-\frac{v_{\varepsilon}}{K}\right) \phi\left(x-\frac{\overline{\mathbf{b}} t}{\varepsilon}, \frac{x}{\varepsilon}, t\right) d \sigma d t= \\
\int_{0}^{T} \int_{\Omega_{\varepsilon}} \operatorname{div}\left(\frac{\mathbf{a}^{\varepsilon}}{\varepsilon}\left(u_{\varepsilon}-\frac{v_{\varepsilon}}{K}\right) \phi\left(x-\frac{\overline{\mathbf{b}} t}{\varepsilon}, \frac{x}{\varepsilon}, t\right)\right) d x d t= \\
\int_{0}^{T} \int_{\Omega_{\varepsilon}}\left(\frac{1}{\varepsilon}\left(u_{\varepsilon}-\frac{v_{\varepsilon}}{K}\right) \frac{\left|\partial \Sigma^{0}\right|}{\left|Y^{0}\right|} \phi\left(x-\frac{\overline{\mathbf{b}} t}{\varepsilon}, \frac{x}{\varepsilon}, t\right)+\right. \\
\quad \mathbf{a}\left(\frac{x}{\varepsilon}\right)\left(\left.\frac{1}{\varepsilon}\left(u_{\varepsilon}-\frac{v_{\varepsilon}}{K}\right) \nabla_{y} \phi\left(x-\frac{\overline{\mathbf{b}} t}{\varepsilon}, y, t\right)\right|_{y=\frac{x}{\varepsilon}}\right. \\
\left.+\left.\nabla\left(u_{\varepsilon}-\frac{v_{\varepsilon}}{K}\right) \phi\left(x-\frac{\overline{\mathbf{b}} t}{\varepsilon}, y, t\right)\right|_{\left.\left.y=\frac{x}{\varepsilon}\right)\right)}\right) d x d t+\mathcal{O}(\varepsilon) \rightarrow \\
\int_{0}^{T} \int_{Y^{0}} \int_{\mathbb{R}^{n}} \operatorname{div}_{y}(q(x, y, t) \phi(x, y, t) \mathbf{a}(y)) d x d y d t, \quad \text { as } \varepsilon \rightarrow 0,
\end{gathered}
$$

where we used that

$$
\left\|\frac{1}{\varepsilon}\left(u_{\varepsilon}-v_{\varepsilon} / K\right)\right\|_{L^{2}\left(\Omega_{\varepsilon} \times(0 ; T)\right)} \leq C \quad \text { and } \quad\left\|\nabla\left(u_{\varepsilon}-v_{\varepsilon} / K\right)\right\|_{L^{2}\left(\Omega_{\varepsilon} \times(0, T)\right)} \leq C .
$$

The surface two-scale limit result (25) follows from (27).

\section{Proof of weak two-scale convergence}

Before proving our main result, Theorem 3, we state and prove a weaker version which relies on the notion of two-scale convergence with drift.

Theorem 11. The sequence $\left\{u_{\varepsilon}, v_{\varepsilon}\right\}$ two-scale converges with drift $(x, t) \rightarrow$ $\left(x-\frac{\overline{\mathbf{b}}}{\varepsilon} t, t\right)$, as $\varepsilon \rightarrow 0$, to the couple $(u(x, t), K u(x, t))$ where $u(x, t)$ is the unique solution of the homogenized problem (14).

The proof of Theorem 11 is divided in five steps, in the spirit of [2].

1. STEP (compactness and choice of the drift)

By virtue of the a priori estimates of section 3.2, Proposition 8 and Corollary 10 imply the existence of a subsequence (still denoted by $\varepsilon$ ) and of limits $\{u, w, q, v\} \in L^{2}\left((0, T) ; H^{1}\left(\mathbb{R}^{n}\right)\right) \times L^{2}\left((0, T) \times \mathbb{R}^{n} ; H^{1}\left(\mathbb{T}^{n}\right)\right)^{2} \times L^{2}\left((0, T) ; H^{1}\left(\mathbb{R}^{n}\right)\right)$ such that

$$
\left\{\begin{array}{l}
u_{\varepsilon} \stackrel{2-\text { drift }}{\longrightarrow} u(x, t), \quad \nabla u_{\varepsilon} \stackrel{2-d r i f t}{\longrightarrow} \nabla u(x, t)+\nabla_{y} w(x, y, t) \\
v_{\varepsilon} \stackrel{2-d r i f t}{\longrightarrow} K u(x, t), \quad \frac{1}{\varepsilon}\left(\frac{v_{\varepsilon}}{K}-u_{\varepsilon}\right) \stackrel{2-\text { drift }}{\longrightarrow} q(x, y, t) .
\end{array}\right.
$$


At this moment the choice of the drift velocity is arbitrary. Nevertheless we now make a choice which will turn out, in the third step, to be the only possible one.

In the absence of chemical reactions (i.e. with homogeneous Neumann boundary condition for $u_{\varepsilon}$ instead of (11)), the drift velocity would be simply $\mathbf{b}_{c}=\left|Y^{0}\right|^{-1} \int_{Y^{0}} \mathbf{b}(y) d y$ (see e.g. [8]). The chemistry term leads to a non-trivial drift which is now defined in agreement with periodic gradient oscillations.

Lemma 4. Let the effective drift $\overline{\mathbf{b}}$ (a constant vector) be given by

$$
\overline{\mathbf{b}}=\left(\left|Y^{0}\right|+\left|\partial \Sigma^{0}\right|_{n-1} K\right)^{-1} \int_{Y^{0}} \mathbf{b}(y) d y .
$$

There exists a periodic solution $\chi_{i}(y) \in H^{1}\left(Y^{0}\right)$ of the following cell problem, $1 \leq i \leq n$,

$$
\begin{gathered}
\mathbf{b}(y) \cdot \nabla \chi_{i}(y)-D \operatorname{div}\left(\nabla\left(\chi_{i}(y)+y_{i}\right)\right)=\bar{b}_{i}-b_{i}(y) \quad \text { in } Y^{0}, \\
D \nabla\left(\chi_{i}(y)+y_{i}\right) \cdot \mathbf{n}=K \bar{b}_{i} \quad \text { on } \partial \Sigma^{0},
\end{gathered}
$$

where $\mathbf{n}(y)$ is the external unit normal on $\partial \Sigma^{0}$. This solution is unique up to an additive constant.

Proof. We check that $\overline{\mathbf{b}}$ is defined precisely so that the compatibility condition (or Fredholm alternative) in (30) is satisfied. We obtain

$$
\int_{Y^{0}} \mathbf{b}(y) d y-\left|Y^{0}\right| \overline{\mathbf{b}}-\int_{\partial \Sigma^{0}} K d \sigma_{y} \overline{\mathbf{b}}=0
$$

where $d \sigma_{y}$ is an element of $(n-1)$-dimensional volume on $\partial \Sigma^{0}$.

2. STEP (determination of the limit function $q$ )

In order to characterize the limit function $q(x, y, t)$, we multiply the equation $\partial_{t} v_{\varepsilon}=\frac{k}{\varepsilon^{2}}\left(u_{\varepsilon}-\frac{v_{\varepsilon}}{K}\right)$ by $\varepsilon \varphi\left(x-\frac{\overline{\mathbf{b}} t}{\varepsilon}, \frac{x}{\varepsilon}, t\right)$, where $\varphi(x, y, t) \in C_{0}^{\infty}\left(\mathbb{R}^{n} \times \mathbb{T}^{n} \times\right.$ $(0, T)$ ), and integrate the resulting expression over $\Omega_{\varepsilon} \times(0, T)$. An integration by parts with respect to time yields

$$
\int_{0}^{T} \int_{\Omega_{\varepsilon}}\left(v_{\varepsilon} \overline{\mathbf{b}} \cdot \nabla_{x} \varphi\left(x-\frac{\overline{\mathbf{b}} t}{\varepsilon}, \frac{x}{\varepsilon}, t\right)-\frac{k}{\varepsilon}\left(u_{\varepsilon}-\frac{v_{\varepsilon}}{K}\right) \varphi\left(x-\frac{\overline{\mathbf{b}} t}{\varepsilon}, \frac{x}{\varepsilon}, t\right)\right) d x d t=\mathcal{O}(\varepsilon),
$$

where we used the notation $\nabla_{x} \varphi\left(x-\frac{\overline{\mathbf{b}} t}{\varepsilon}, \frac{x}{\varepsilon}, t\right)=\left.\nabla_{x} \varphi\left(x-\frac{\overline{\mathbf{b}} t}{\varepsilon}, y, t\right)\right|_{y=x / \varepsilon}$ and $\nabla_{y} \varphi\left(x-\frac{\overline{\mathbf{b}} t}{\varepsilon}, \frac{x}{\varepsilon}, t\right)=\left.\nabla_{y} \varphi\left(x-\frac{\overline{\mathbf{b}} t}{\varepsilon}, y, t\right)\right|_{y=x / \varepsilon}$. Passing to the two-scale 
limit with drift and bearing in mind that the two-scale limit of $v_{\varepsilon}$ is equal to $K u(x, t)$, we obtain

$$
\int_{0}^{T} \int_{\mathbb{R}^{n}} \int_{Y^{0}}\left(K u(x, t) \overline{\mathbf{b}} \cdot \nabla_{x} \varphi(x, y, t)-k q(x, y, t) \varphi(x, y, t)\right) d x d y d t=0
$$

Therefore,

$$
q(x, y, t)=q(x, t)=-\frac{K}{k} \overline{\mathbf{b}} \cdot \nabla_{x} u(x, t) .
$$

\section{STEP (determination of the limit function $w$ )}

In order to characterize the limit function $w(x, y, t)$, we choose again a test function as before:

$$
\varphi_{\varepsilon}=\varepsilon \varphi\left(x-\frac{\overline{\mathbf{b}} t}{\varepsilon}, \frac{x}{\varepsilon}, t\right) .
$$

Substituting it in problem (10)-(12) yields

$$
\begin{gathered}
\int_{0}^{T} \int_{\Omega_{\varepsilon}}\left\{u_{\varepsilon} \overline{\mathbf{b}} \cdot \nabla_{x} \varphi\left(x-\frac{\overline{\mathbf{b}} t}{\varepsilon}, \frac{x}{\varepsilon}, t\right)+\mathbf{b}\left(\frac{x}{\varepsilon}\right) \cdot \nabla u_{\varepsilon} \varphi\left(x-\frac{\overline{\mathbf{b}} t}{\varepsilon}, \frac{x}{\varepsilon}, t\right)+\right. \\
\left.D \nabla u_{\varepsilon} \nabla_{y} \varphi\left(x-\frac{\overline{\mathbf{b}} t}{\varepsilon}, \frac{x}{\varepsilon}, t\right)\right\} d x d t+\varepsilon \int_{0}^{T} \int_{\partial \Omega_{\varepsilon}} \frac{k}{\varepsilon}\left(u_{\varepsilon}-\frac{v_{\varepsilon}}{K}\right) \varphi\left(x-\frac{\overline{\mathbf{b}} t}{\varepsilon}, \frac{x}{\varepsilon}, t\right) d \sigma d t \\
=\mathcal{O}(\varepsilon) .
\end{gathered}
$$

Passing to the two-scale limit with drift gives us the cell problem

$$
\begin{gathered}
\int_{0}^{T} \int_{\mathbb{R}^{n}} \int_{Y^{0}}\left\{u(x, t) \overline{\mathbf{b}} \cdot \nabla_{x} \varphi(x, y, t)+\mathbf{b}(y) \cdot\left(\nabla_{x} u(x, t)+\nabla_{y} w(x, y, t)\right) \varphi(x, y, t)+\right. \\
\left.D\left(\nabla_{x} u(x, t)+\nabla_{y} w(x, y, t)\right) \cdot \nabla_{y} \varphi(x, y, t)\right\} d x d y d t+ \\
\int_{0}^{T} \int_{\mathbb{R}^{n}} \int_{\partial \Sigma^{0}} k q(x, y, t) \varphi(x, y, t) d x d \sigma_{y} d t=0 .
\end{gathered}
$$

As in classical two-scale convergence, problem (34) leads to the following differential problem for $w$, valid a.e. on $(0, T) \times \mathbb{R}^{n}$ :

$$
\begin{gathered}
-D \operatorname{div}_{y}\left(\nabla_{x} u(x, t)+\nabla_{y} w(x, y, t)\right)+\mathbf{b}(y) \cdot\left(\nabla_{x} u(x, t)+\nabla_{y} w(x, y, t)\right)= \\
\overline{\mathbf{b}} \cdot \nabla_{x} u(x, t) \quad \text { in } Y^{0} \\
w \text { is } Y \text {-periodic in } y \\
-D\left(\nabla_{x} u(x, t)+\nabla_{y} w(x, y, t)\right) \cdot \mathbf{n}=k q(x, t)=-K \nabla_{x} u(x, t) \quad \text { on } \partial \Sigma^{0} .
\end{gathered}
$$

At this point it is crucial to have chosen the drift $\overline{\mathbf{b}}$ defined by (29), otherwise (37) would have no solution but the trivial one. Finally, we conclude that 
the function $w(x, y, t)$ is given by the following separation of fast and slow variables formula:

$$
w(x, y, t)=\chi(y) \cdot \nabla_{x} u(x, t),
$$

with $\chi(y)$ of components $\chi_{i}$ solving problem (30).

4. STEP (determination of the homogenized equation)

In this step we test problem (10)-(12) by $\hat{\phi}(x, t)=\phi\left(x-\frac{\overline{\mathbf{b}} t}{\varepsilon}, t\right)$, with $\phi \in C_{0}^{\infty}\left(\mathbb{R}^{n} \times[0, T)\right)$, implying that $\phi(x, T)=0$. Also, we use the symbol $\widehat{\partial_{t} \phi}$ for $\left.\partial_{t} \phi(z, t)\right|_{z=x-\overline{\mathbf{b}} t / \varepsilon}$. Note that

$$
\partial_{t} \hat{\phi}(x, t)=\widehat{\partial_{t} \phi}(x, t)-\frac{\overline{\mathbf{b}}}{\varepsilon} \cdot \nabla_{x} \hat{\phi}(x, t) .
$$

We get

$$
\begin{aligned}
\int_{0}^{T} \int_{\Omega_{\varepsilon}}\left\{u_{\varepsilon} \frac{\overline{\mathbf{b}}-\mathbf{b}\left(\frac{x}{\varepsilon}\right)}{\varepsilon} \cdot \nabla_{x} \hat{\phi}-u_{\varepsilon} \widehat{\partial_{t} \phi}\right\} d x d t+\int_{0}^{T} \int_{\partial \Omega_{\varepsilon}} v_{\varepsilon}(x, t) \overline{\mathbf{b}} \cdot \nabla_{x} \hat{\phi} d \sigma d t- \\
\\
\int_{\Omega_{\varepsilon}} u^{0}(x) \phi(x, 0) d x+D \int_{0}^{T} \int_{\Omega_{\varepsilon}} \nabla u_{\varepsilon} \cdot \nabla_{x} \hat{\phi} d x d t- \\
\varepsilon \int_{0}^{T} \int_{\partial \Omega_{\varepsilon}} v_{\varepsilon}(x, t) \widehat{\partial_{t} \phi} d \sigma d t-\varepsilon \int_{\partial \Omega_{\varepsilon}} v^{0}(x) \phi(x, 0) d \sigma=0 .
\end{aligned}
$$

Next we introduce the auxiliary vector function $\psi$ by

$$
\left\{\begin{array}{l}
\Delta \psi_{i}(y)=b_{i}(y)-\bar{b}_{i} \text { on } Y^{0} \\
\frac{\partial \psi_{i}}{\partial \mathbf{n}}=K \bar{b}_{i} \quad \text { on } \partial \Sigma^{0} \\
\psi_{i} \quad \text { is } 1-\text { periodic. }
\end{array}\right.
$$

Then for $\psi^{\varepsilon}(x)=\psi(x / \varepsilon)$ we have

$$
\left\{\begin{array}{l}
\varepsilon^{2} \Delta \psi_{i}^{\varepsilon}(x)=b_{i}\left(\frac{x}{\varepsilon}\right)-\bar{b}_{i} \text { in } \Omega_{\varepsilon} \\
\varepsilon \frac{\partial \psi_{i}^{\varepsilon}}{\partial \mathbf{n}}=K \bar{b}_{i} \quad \text { on } \partial \Omega_{\varepsilon}
\end{array}\right.
$$


Furthermore, we have the following integral identity

$$
\begin{array}{r}
\int_{0}^{T} \int_{\Omega_{\varepsilon}} u_{\varepsilon} \frac{\overline{\mathbf{b}}-\mathbf{b}\left(\frac{x}{\varepsilon}\right)}{\varepsilon} \cdot \nabla_{x} \hat{\phi} d x d t+\int_{0}^{T} \int_{\partial \Omega_{\varepsilon}} v_{\varepsilon}(x, t) \overline{\mathbf{b}} \cdot \nabla_{x} \hat{\phi} d \sigma d t= \\
-\int_{0}^{T} \int_{\Omega_{\varepsilon}} \varepsilon \sum_{i=1}^{n} \Delta \psi_{i}^{\varepsilon} \partial_{x_{i}} \hat{\phi} u_{\varepsilon} d x d t+\int_{0}^{T} \int_{\partial \Omega_{\varepsilon}} v_{\varepsilon}(x, t) \overline{\mathbf{b}} \cdot \nabla_{x} \hat{\phi} d \sigma d t= \\
K \int_{0}^{T} \int_{\partial \Omega_{\varepsilon}}\left(\frac{v_{\varepsilon}}{K}-u_{\varepsilon}\right) \overline{\mathbf{b}} \cdot \nabla_{x} \hat{\phi} d \sigma d t+\int_{0}^{T} \int_{\Omega_{\varepsilon}} \varepsilon \sum_{i=1}^{n} \nabla \psi_{i}^{\varepsilon} \cdot \nabla\left(\partial_{x_{i}} \hat{\phi} u_{\varepsilon}\right) d x d t .
\end{array}
$$

Inserting (42) into (39) gives

$$
\begin{gathered}
K \varepsilon \int_{0}^{T} \int_{\partial \Omega_{\varepsilon}} \frac{1}{\varepsilon}\left(\frac{v_{\varepsilon}}{K}-u_{\varepsilon}\right) \overline{\mathbf{b}} \cdot \nabla_{x} \hat{\phi} d \sigma d t+\int_{0}^{T} \int_{\Omega_{\varepsilon}} \varepsilon \sum_{i=1}^{n} \nabla \psi_{i}^{\varepsilon} \cdot \nabla\left(\partial_{x_{i}} \hat{\phi} u_{\varepsilon}\right) d x d t- \\
\int_{0}^{T} \int_{\Omega_{\varepsilon}} u_{\varepsilon} \widehat{\partial_{t} \phi} d x d t-\int_{\Omega_{\varepsilon}} u^{0}(x) \phi(x, 0) d x+D \int_{0}^{T} \int_{\Omega_{\varepsilon}} \nabla u_{\varepsilon} \cdot \nabla_{x} \hat{\phi} d x d t- \\
\quad \int_{0}^{T} \int_{\partial \Omega_{\varepsilon}} v_{\varepsilon}(x, t) \widehat{\partial_{t} \phi} d \sigma d t-\varepsilon \int_{\partial \Omega_{\varepsilon}} v^{0}(x) \phi(x, 0) d \sigma=0 .
\end{gathered}
$$

Passing to the two-scale limit with drift $(x, t) \rightarrow(x-\overline{\mathbf{b}} t / \varepsilon, t)$ in the last relation is now straightforward. For the comfort of the reader, we do it term by term:

$$
\begin{gathered}
\lim _{\varepsilon \rightarrow 0} \int_{0}^{T} \int_{\Omega_{\varepsilon}} u_{\varepsilon} \widehat{\partial_{t} \phi} d x d t=\left|Y^{0}\right| \int_{0}^{T} \int_{\mathbb{R}^{n}} u(x, t) \partial_{t} \phi(x, t) d x d t \\
\lim _{\varepsilon \rightarrow 0} \varepsilon \int_{0}^{T} \int_{\partial \Omega_{\varepsilon}} v_{\varepsilon}(x, t) \widehat{\partial_{t} \phi} d \sigma d t=\left|\partial \Sigma^{0}\right|_{n-1} \int_{0}^{T} \int_{\mathbb{R}^{n}} K u(x, t) \partial_{t} \phi(x, t) d x d t, \\
\lim _{\varepsilon \rightarrow 0} D \int_{0}^{T} \int_{\Omega_{\varepsilon}} \nabla u_{\varepsilon} \cdot \nabla_{x} \hat{\phi} d x d t=D \int_{0}^{T} \int_{\mathbb{R}^{n}} \sum_{i=1}^{n}\left(\left|Y^{0}\right| \partial_{x_{i}} u(x, t)+\right. \\
\left.\sum_{j=1}^{n} \partial_{x_{j}} u(x, t) \int_{Y^{0}} \frac{\partial \chi_{j}(y)}{\partial y_{i}} d y\right) \partial_{x_{i}} \phi(x, t) d x d t
\end{gathered}
$$




$$
\begin{gathered}
\lim _{\varepsilon \rightarrow 0} K \varepsilon \int_{0} \int_{\partial \Omega_{\varepsilon}} \frac{1}{\varepsilon}\left(\frac{v_{\varepsilon}}{K}-u_{\varepsilon}\right) \overline{\mathbf{b}} \cdot \nabla_{x} \hat{\phi} d \sigma d t= \\
\int_{0}^{T} \int_{\mathbb{R}^{n}}\left|\partial \Sigma^{0}\right|_{n-1} \frac{K^{2}}{k} \overline{\mathbf{b}} \otimes \overline{\mathbf{b}} \nabla_{x} u \nabla_{x} \phi d x d t, \\
\lim _{\varepsilon \rightarrow 0} \int_{0}^{T} \int_{\Omega_{\varepsilon}} \varepsilon \sum_{i=1}^{n} \nabla \psi_{i}^{\varepsilon} \cdot \nabla\left(\partial_{x_{i}} \hat{\phi} u_{\varepsilon}\right) d x d t= \\
\int_{0}^{T} \int_{\mathbb{R}^{n}} \sum_{i, j=1}^{n} \frac{\partial^{2} \phi}{\partial x_{i} x_{j}} u(x, t)\left(\int_{Y^{0}} \frac{\partial \psi_{i}}{\partial y_{j}} d y\right) d x d t+ \\
\int_{0}^{T} \int_{\mathbb{R}^{n}} \int_{Y^{0}} \sum_{i, j=1}^{n} \partial_{x_{i}} \phi(x, t) \frac{\partial \psi_{i}(y)}{\partial y_{j}}\left(\partial_{x_{j}} u+\partial_{x_{\ell}} u \sum_{\ell=1}^{n} \frac{\partial \chi_{\ell}(y)}{\partial y_{j}}\right) d y d x d t .
\end{gathered}
$$

It is now time to introduce the homogenized matrix which for simplicity we decompose as a sum of elementary matrices. The first one linked to adsorption/desorption reactions, transported by the drift velocity, is calculated in (47) and given by

$$
\bar{A}_{1}=\frac{K^{2}}{k}\left|\partial \Sigma^{0}\right|_{n-1} \overline{\mathbf{b}} \otimes \overline{\mathbf{b}}=\left[\frac{K^{2}}{k}\left|\partial \Sigma^{0}\right|_{n-1} \bar{b}_{i} \bar{b}_{j}\right] .
$$

The second one, related to advection-diffusion and chemistry, is calculated in (46)-(48) and given by

$$
\left[\bar{A}_{2}\right]_{i j}=D \int_{Y^{0}}\left(\delta_{i j}+\frac{\partial \chi_{i}(y)}{\partial y_{j}}\right) d y+\sum_{\ell=1}^{n} \int_{Y^{0}} \frac{\partial \chi_{j}(y)}{\partial y_{\ell}} \frac{\partial \psi_{i}(y)}{\partial y_{\ell}} d y .
$$

Remark that only the symmetric part of the homogenized matrix appears in the homogenized equation: $\bar{A}_{1}$ is already symmetric but $\bar{A}_{2}$ is not and should be symmetrized. The effective or homogenized matrix is thus defined by

$$
A^{*}=\bar{A}_{1}+\frac{1}{2}\left(\bar{A}_{2}+\bar{A}_{2}^{\mathcal{T}}\right) .
$$

Then after inserting the limits (44)-(48) into the variational equation (43) we conclude that the limit function $u(x, t)$ solves the problem

$$
\begin{gathered}
\left(\left|Y^{0}\right|+K\left|\partial \Sigma^{0}\right|_{n-1}\right) \partial_{t} u=\operatorname{div}_{x}\left(A^{*} \nabla_{x} u\right) \quad \text { in } \mathbb{R}^{n} \times(0, T), \\
u(x, 0)=\frac{\left|Y^{0}\right| u^{0}(x)+\left|\partial \Sigma^{0}\right|_{n-1} v^{0}(x)}{\left|Y^{0}\right|+K\left|\partial \Sigma^{0}\right|_{n-1}} \text { in } \mathbb{R}^{n} .
\end{gathered}
$$

It remains to prove that the matrix $A^{*}$ is positive definite and establish uniqueness of the limit function. 
5. STEP (properties of the effective matrices and uniqueness)

Clearly, the matrix $\bar{A}_{1}$, given by (49), is symmetric and non-negative, i.e. $\bar{A}_{1} \xi \cdot \xi \geq 0$ for any $\xi \in \mathbb{R}^{n}$. Furthermore, we have

Lemma 5. The matrix $\bar{A}_{2}$, given by (50), is positive definite and satisfies

$$
\left[\bar{A}_{2}\right]_{i j}=D \int_{Y^{0}}\left(\nabla_{y} \chi_{i}(y)+e_{i}\right) \cdot\left(\nabla_{y} \chi_{j}(y)+e_{j}\right) d y+\int_{Y^{0}} \mathbf{b}(y) \cdot \nabla_{y} \chi_{i}(y) \chi_{j}(y) d y .
$$

Finally, $A^{*}$ is also positive definite, equivalently defined by

$$
A^{*}=\frac{K^{2}}{k}\left|\partial \Sigma^{0}\right|_{n-1} \overline{\mathbf{b}} \otimes \overline{\mathbf{b}}+D \int_{Y^{0}}\left(\mathbf{I}+\nabla_{y} \chi(y)\right)\left(\mathbf{I}+\nabla_{y} \chi(y)\right)^{\mathcal{T}} d y .
$$

Proof. First we test problem (40) for $\psi_{i}$ by $\chi_{j}$. The second term on the right-hand side of (50) becomes

$$
\sum_{\ell=1}^{n} \int_{Y^{0}} \frac{\partial \chi_{j}(y)}{\partial y_{\ell}} \frac{\partial \psi_{i}(y)}{\partial y_{\ell}} d y=\int_{Y}\left(\bar{b}_{i}-b_{i}(y)\right) \chi_{j}(y) d y+K \int_{\partial \Sigma^{0}} \bar{b}_{i} \chi_{j}(y) d \sigma .
$$

Next we multiply the equation (30) for $\chi_{i}$ by $\chi_{j}(y)$ and integrate the resulting relation over $Y^{0}$. This yields a formula for the first term on the right-hand side of $(50)$

$$
\begin{gathered}
\int_{Y}\left(\bar{b}_{i}-b_{i}(y)\right) \chi_{j}(y) d y+K \int_{\partial \Sigma^{0}} \bar{b}_{i} \chi_{j}(y) d \sigma=\int_{Y^{0}} \mathbf{b}(y) \cdot \nabla_{y} \chi_{i}(y) \chi_{j}(y) d y+ \\
D \int_{Y^{0}}\left(e_{i}+\nabla_{y} \chi_{i}(y)\right) \cdot \nabla_{y} \chi_{j}(y) d y=-D \int_{Y^{0}}\left(\delta_{i j}+\frac{\partial \chi_{i}(y)}{\partial y_{j}}\right) d y+ \\
\int_{Y^{0}} \mathbf{b}(y) \cdot \nabla_{y} \chi_{i}(y) \chi_{j}(y) d y+D \int_{Y^{0}}\left(\nabla_{y} \chi_{i}(y)+e_{i}\right) \cdot\left(\nabla_{y} \chi_{j}(y)+e_{j}\right) d y
\end{gathered}
$$

Identities (55)-(56) imply (53). Since $\mathbf{b}(y)$ is solenoidal and its normal component is equal to zero at $\partial \Sigma^{0}$, we find easily that the matrix $\left\{\int_{Y^{0}} \mathbf{b}(y)\right.$. $\left.\nabla_{y} \chi_{i}(y) \chi_{j}(y) d y\right\}$ is skew-symmetric:

$$
-\int_{Y^{0}} \mathbf{b}(y) \cdot \nabla_{y} \chi_{j}(y) \chi_{i}(y) d y=\int_{Y^{0}} \mathbf{b}(y) \cdot \nabla_{y} \chi_{i}(y) \chi_{j}(y) d y, \quad i, j=1, \ldots, n .
$$

The remaining part of Lemma (5) follows immediately.

As a consequence, the uniqueness of the homogenized solution $u(t, x)$ is obvious. Thus the entire sequence $\left\{u_{\varepsilon}, v_{\varepsilon}\right\}$ is converging. 


\section{Strong convergence (proof of Theorem 3)}

This section is devoted to the proof of Theorem 3, i.e. it improves on Theorem 11 by replacing the weak two-scale convergence (with drift) with a strong convergence result. Namely, we are going to show that in the moving coordinates $(x, t) \rightarrow(x-(\overline{\mathbf{b}} / \varepsilon) t, t)$ the functions $u_{\varepsilon}$ and $v_{\varepsilon}$ converge strongly to the limit functions $u(x, t)$ and $K u(x, t)$, respectively, or equivalently that

$$
\lim _{\varepsilon \rightarrow 0}\left\|u_{\varepsilon}(x, t)-u\left(x-\frac{\overline{\mathbf{b}} t}{\varepsilon}, t\right)\right\|_{L^{2}\left(\Omega_{\varepsilon} \times(0, T)\right)}=0 .
$$

We start with the case of well-prepared initial data, i.e. the initial data are at the equilibrium isotherm. Our first result is the following

Proposition 12. Let $v^{0}(x)=K u^{0}(x) \in H^{1}\left(\mathbb{R}^{n}\right)$ (i.e. initial data at the isotherm). Then $u_{\varepsilon}(x, t) \chi_{\Omega_{\varepsilon}}$ strongly two-scale converges with drift $(x, t) \rightarrow$ $\left(x-\frac{\overline{\mathbf{b}} t}{\varepsilon}, t\right)$ in $\mathbb{R}^{n} \times(0, T)$ towards $\chi_{Y^{0}}(y) u(x, t)$. Similarly, $v_{\varepsilon}(x, t) \chi_{\Omega_{\varepsilon}}$ strongly two-scale converges with drift towards $K_{\chi_{Y^{0}}}(y) u(x, t)$. In particular,

$$
\int_{0}^{T} \int_{\Omega_{\varepsilon}}\left|u_{\varepsilon}(x, t)-u\left(x-\frac{\overline{\mathbf{b}}}{\varepsilon} t, t\right)\right|^{2} d x d t \underset{\varepsilon \rightarrow 0}{\longrightarrow} 0 .
$$

Proof. We start by integrating the energy equality (18) in time variable over the interval $(0, t)$. This yields

$$
\begin{gathered}
\frac{1}{2}\left[\left\|u_{\varepsilon}(t)\right\|_{L^{2}\left(\Omega_{\varepsilon}\right)}^{2}+\frac{\varepsilon}{K}\left\|v_{\varepsilon}(t)\right\|_{L^{2}\left(\partial \Omega_{\varepsilon}\right)}^{2}\right]+\int_{0}^{t} \int_{\Omega_{\varepsilon}} D \nabla u_{\varepsilon}(s) \cdot \nabla u_{\varepsilon}(s) d x d s+ \\
\int_{0}^{t} \frac{\varepsilon k}{\varepsilon^{2}} \int_{\partial \Omega_{\varepsilon}}\left(u_{\varepsilon}(s)-\frac{v_{\varepsilon}(s)}{K}\right)^{2} d \sigma d s=\frac{1}{2}\left[\left\|u^{0}\right\|_{L^{2}\left(\Omega_{\varepsilon}\right)}^{2}+\frac{\varepsilon}{K}\left\|v^{0}\right\|_{L^{2}\left(\partial \Omega_{\varepsilon}\right)}^{2}\right] .
\end{gathered}
$$

Since we expect the family $\left\{u_{\varepsilon}, v_{\varepsilon}\right\}$ to be compact only in the product space $L^{2}\left((0, T) \times \Omega_{\varepsilon}\right)$, it is out of reach to claim convergence of these functions for a fixed value of $t$. To circumvent this difficulty, we integrate formula (58) in temporal variable once again. The resulting formula reads

$$
\begin{gathered}
\frac{1}{2} \int_{0}^{T}\left[\left\|u_{\varepsilon}(t)\right\|_{L^{2}\left(\Omega_{\varepsilon}\right)}^{2}+\frac{\varepsilon}{K}\left\|v_{\varepsilon}(t)\right\|_{L^{2}\left(\partial \Omega_{\varepsilon}\right)}^{2}\right] d t+\int_{0}^{T} \int_{0}^{t} \int_{\Omega_{\varepsilon}} D \nabla u_{\varepsilon}(s) \cdot \nabla u_{\varepsilon}(s) d x d s d t+ \\
\frac{k}{\varepsilon} \int_{0}^{T} \int_{0}^{t} \int_{\partial \Omega_{\varepsilon}}\left(u_{\varepsilon}(s)-\frac{v_{\varepsilon}(s)}{K}\right)^{2} d \sigma d s d t=\frac{T}{2}\left[\left\|u^{0}\right\|_{L^{2}\left(\Omega_{\varepsilon}\right)}^{2}+\frac{\varepsilon}{K}\left\|v^{0}\right\|_{L^{2}\left(\partial \Omega_{\varepsilon}\right)}^{2}\right] .
\end{gathered}
$$


Using the two-scale convergence results of the previous section and taking into account the lower semicontinuity of the corresponding norms with respect to the two-scale and weak convergence (see [2] if necessary), we have

$$
\begin{aligned}
& \liminf _{\varepsilon \rightarrow 0} \int_{0}^{T}\left[\left\|u_{\varepsilon}(t)\right\|_{L^{2}\left(\Omega_{\varepsilon}\right)}^{2}+\frac{\varepsilon}{K}\left\|v_{\varepsilon}(t)\right\|_{L^{2}\left(\partial \Omega_{\varepsilon}\right)}^{2}\right] d t \geq\left|Y^{0}\right|\|u\|_{L^{2}\left(\mathbb{R}^{n} \times(0, T)\right)}^{2}+ \\
& \left|\partial \Sigma^{0}\right|_{n-1} K\|u\|_{L^{2}\left(\mathbb{R}^{n} \times(0, T)\right)}^{2}=\left(\left|Y^{0}\right|+\left|\partial \Sigma^{0}\right|_{n-1} K\right)\|u\|_{L^{2}\left(\mathbb{R}^{n} \times(0, T)\right)}^{2} .
\end{aligned}
$$

By the same arguments,

$$
\begin{gathered}
\liminf _{\varepsilon \rightarrow 0} \int_{0}^{T} \int_{0}^{t} \int_{\Omega_{\varepsilon}} D \nabla u_{\varepsilon}(x, s) \cdot \nabla u_{\varepsilon}(x, s) d x d s d t \geq \\
\int_{0}^{T} \int_{0}^{t} \int_{\mathbb{R}^{n} Y^{0}} \int_{x} D\left|\nabla_{x} u(x, s)+\nabla_{y} \chi(y) \nabla_{x} u(x, s)\right|^{2} d y d x d s d t
\end{gathered}
$$

and

$$
\begin{gathered}
\liminf _{\varepsilon \rightarrow 0} \frac{k}{\varepsilon} \int_{0}^{T} \int_{0}^{t} \int_{\partial \Omega_{\varepsilon}}\left(u_{\varepsilon}(x, s)-\frac{v_{\varepsilon}(x, s)}{K}\right)^{2} d \sigma d s d t \geq \\
k\left|\partial \Sigma^{0}\right|_{n-1} \int_{0}^{T} \int_{0}^{t} \int_{\mathbb{R}^{n}}\left|\frac{K}{k} \overline{\mathbf{b}} \cdot \nabla_{x} u(x, s)\right|^{2} d x d s d t .
\end{gathered}
$$

Passing to the limit on the right hand side of (59), we get

$\lim _{\varepsilon \rightarrow 0} \frac{T}{2}\left[\left\|u^{0}\right\|_{L^{2}\left(\Omega_{\varepsilon}\right)}^{2}+\frac{\varepsilon}{K}\left\|v^{0}\right\|_{L^{2}\left(\partial \Omega_{\varepsilon}\right)}^{2}\right]=\frac{T}{2}\left|Y^{0}\right|\left\|u^{0}\right\|_{L^{2}\left(\mathbb{R}^{n}\right)}^{2}+\frac{T}{2 K}\left|\partial \Sigma^{0}\right|_{n-1}\left\|v^{0}\right\|_{L^{2}\left(\mathbb{R}^{n}\right)}^{2}$.

Our next aim is to compute the energy of the limit equation. Multiplying equation (51) by $u(x, s)$ and integrating over $\mathbb{R}^{n} \times(0, t)$ and then once again in variable $t$ over the interval $(0, T)$, after straightforward transformations we obtain

$$
\begin{gathered}
\frac{1}{2}\left(\left|Y^{0}\right|+\left|\partial \Sigma^{0}\right|_{n-1} K\right)\|u\|_{L^{2}\left(\mathbb{R}^{n} \times(0, T)\right)}^{2}+\int_{0}^{T} \int_{0}^{t} \int_{\mathbb{R}^{n}} A^{*} \nabla u(x, s) \cdot \nabla u(x, s) d x d s d t= \\
\frac{T}{2\left(\left|Y^{0}\right|+K\left|\partial \Sigma^{0}\right|_{n-1}\right)}\left\|\left(\left|Y^{0}\right| u^{0}+\left|\partial \Sigma^{0}\right| v^{0}\right)\right\|_{L^{2}\left(\mathbb{R}^{n}\right)}^{2}
\end{gathered}
$$

Due to (54) the second integral on the left hand side can be rearranged as follows

$$
\int_{0}^{T} \int_{0}^{t} \int_{\mathbb{R}^{n}} A^{*} \nabla u(x, s) \cdot \nabla u(x, s) d x d s d t
$$




$$
\begin{gathered}
=\int_{0}^{T} \int_{0}^{t} \int_{\mathbb{R}^{n}} \int_{Y^{0}} D\left|\nabla_{x} u(x, s)+\nabla_{y} \chi(y) \nabla_{x} u(x, s)\right|^{2} d y d x d s d t \\
\quad+\int_{0}^{T} \int_{0}^{t} \int_{\mathbb{R}^{n}} \frac{K^{2}}{k}\left|\partial \Sigma^{0}\right|_{n-1}\left(\overline{\mathbf{b}} \cdot \nabla_{x} u(x, t)\right)^{2} d x d s d t .
\end{gathered}
$$

Because of the energy equality (63) for the homogenized problem and the lower semicontinuity of the terms in the energy equality (59) for the microscopic problem, we conclude that the norm convergence is valid if and only if we have

$$
\begin{gathered}
\left(\left|Y^{0}\right|+K\left|\partial \Sigma^{0}\right|_{n-1}\right)^{-1}\left\|\left(\left|Y^{0}\right| u^{0}+\left|\partial \Sigma^{0}\right|_{n-1} v^{0}\right)\right\|_{L^{2}\left(\mathbb{R}^{n}\right)}^{2}= \\
\left(\left|Y^{0}\right|\left\|u^{0}\right\|_{L^{2}\left(\mathbb{R}^{n}\right)}^{2}+K^{-1}\left|\partial \Sigma^{0}\right|_{n-1}\left\|v^{0}\right\|_{L^{2}\left(\mathbb{R}^{n}\right)}^{2}\right) .
\end{gathered}
$$

After a simple calculation, we find out that (64) is equivalent to $\| u^{0} \sqrt{K}-$ $v^{0} / \sqrt{K} \|_{L^{2}\left(\mathbb{R}^{n}\right)}^{2}=0$. Hence under our assumptions on well prepared initial data, we have

$$
\begin{gathered}
\lim _{\varepsilon \rightarrow 0}\left\|u_{\varepsilon}\right\|_{L^{2}\left(\Omega_{\varepsilon} \times(0, T)\right)}^{2}=\left|Y^{0}\right|\|u\|_{L^{2}\left(\mathbb{R}^{n} \times(0, T)\right)}^{2}=\left\|\chi_{Y^{0}}(y) u(x, t)\right\|_{L^{2}\left(Y \times \mathbb{R}^{n} \times(0, T)\right)}^{2} \\
\lim _{\varepsilon \rightarrow 0} \varepsilon\left\|v_{\varepsilon}\right\|_{L^{2}\left(\partial \Omega_{\varepsilon} \times(0, T)\right)}^{2}=K^{2}\left|\partial \Sigma^{0}\right|_{n-1}\|u\|_{L^{2}\left(\mathbb{R}^{n} \times(0, T)\right)}^{2}
\end{gathered}
$$

which is equivalent to the desired strong two-scale convergence (see [2]).

In order to prove the strong convergence result for $u_{\varepsilon}$ in the case of arbitrary initial conditions $u^{0} \in L^{2}\left(\mathbb{R}^{n}\right)$ and $v^{0} \in H^{1}\left(\mathbb{R}^{n}\right)$, and thus to finish the proof of Theorem 3, we consider problem (10)-(12) on subintervals $t \in(\delta, T)$ with small positive $\delta$. For non-consistent initial conditions the solution $\left(u_{\varepsilon}, v_{\varepsilon}\right)$ contains an initial layer term which makes a nontrivial contribution to the energy. By restricting problem (10) on subinterval $(\delta, T)$, we make this contribution negligible for small $\delta$.

We proceed with rigorous arguments. Assume that for a subsequence (still denoted by $\varepsilon$ ) there is a lack of energy continuity, i.e.,

$$
\lim _{\varepsilon \rightarrow 0}\left\|u_{\varepsilon}\right\|_{L^{2}\left(\Omega_{\varepsilon} \times(0, T)\right)}^{2}>\left|Y^{0}\right|\|u\|_{L^{2}\left(\mathbb{R}^{n} \times(0, T)\right)}^{2} .
$$

Then, since $\left\|u_{\varepsilon}(t)\right\|_{L^{2}\left(\Omega_{\varepsilon}\right)}^{2} \leq C$ uniformly in time by Lemma 1 , there is $\delta_{0}>0$ such that for any $\delta, 0<\delta<\delta_{0}$, we have

$$
\liminf _{\varepsilon \rightarrow 0}\left\|u_{\varepsilon}\right\|_{L^{2}\left(\Omega_{\varepsilon} \times(\delta, T)\right)}^{2}>\left|Y^{0}\right|\|u\|_{L^{2}\left(\mathbb{R}^{n} \times(0, T)\right)}^{2} .
$$

It follows from (18) and Lemmata 2 and 3 that there exist a constant $c$ (depending on the initial data but not on $\delta_{0}$ ) and a sequence $\delta_{\varepsilon}$ such that $\delta_{0} / 2 \leq \delta_{\varepsilon} \leq \delta_{0}$, and

$$
\left\|u_{\varepsilon}\left(\cdot, \delta_{\varepsilon}\right)\right\|_{H^{1}\left(\Omega_{\varepsilon}\right)}^{2} \leq \frac{c}{\delta_{0}}, \quad\left\|v_{\varepsilon}\left(\cdot, \delta_{\varepsilon}\right)\right\|_{H^{1}\left(\Omega_{\varepsilon}\right)}^{2} \leq \frac{c}{\delta_{0}}, \quad\left\|K u_{\varepsilon}\left(\cdot, \delta_{\varepsilon}\right)-v_{\varepsilon}\left(\cdot, \delta_{\varepsilon}\right)\right\|_{L^{2}\left(\Omega_{\varepsilon}\right)}^{2} \leq \frac{c \varepsilon^{2}}{\delta_{0}} .
$$


Taking, if necessary, a subsequence, one can assume that $\delta_{\varepsilon}$ converges to some $\bar{\delta}, \delta_{0} / 2 \leq \bar{\delta} \leq \delta_{0}$. Consider two auxiliary problems

$$
\left\{\begin{array}{cc}
\partial_{t} u_{1, \varepsilon}+\frac{1}{\varepsilon} \mathbf{b}\left(\frac{x}{\varepsilon}\right) \cdot \nabla u_{1, \varepsilon}-\Delta u_{1, \varepsilon}=0 & \text { in } \Omega_{\varepsilon} \times\left(\delta_{\varepsilon}, T\right), \\
-\frac{1}{\varepsilon} \frac{\partial u_{1, \varepsilon}}{\partial \mathbf{n}}=\partial_{t} v_{1, \varepsilon}=\frac{k}{\varepsilon^{2}}\left(u_{1, \varepsilon}-\frac{v_{1, \varepsilon}}{K}\right) & \text { on } \partial \Omega_{\varepsilon} \times\left(\delta_{\varepsilon}, T\right), \\
u_{1, \varepsilon}\left(x, \delta_{\varepsilon}\right)=u_{\varepsilon}\left(x, \delta_{\varepsilon}\right), \quad v_{1, \varepsilon}\left(x, \delta_{\varepsilon}\right)=K u_{\varepsilon}\left(x, \delta_{\varepsilon}\right) ;
\end{array}\right.
$$

and

$$
\left\{\begin{array}{cc}
\partial_{t} u_{2, \varepsilon}+\frac{1}{\varepsilon} \mathbf{b}\left(\frac{x}{\varepsilon}\right) \cdot \nabla u_{2, \varepsilon}-\Delta u_{2, \varepsilon}=0 & \text { in } \Omega_{\varepsilon} \times\left(\delta_{\varepsilon}, T\right), \\
-\frac{1}{\varepsilon} \frac{\partial u_{2, \varepsilon}}{\partial \mathbf{n}}=\partial_{t} v_{2, \varepsilon}=\frac{k}{\varepsilon^{2}}\left(u_{2, \varepsilon}-\frac{v_{2, \varepsilon}}{K}\right) & \text { on } \partial \Omega_{\varepsilon} \times\left(\delta_{\varepsilon}, T\right), \\
u_{2, \varepsilon}\left(x, \delta_{\varepsilon}\right)=0, \quad v_{2, \varepsilon}\left(x, \delta_{\varepsilon}\right)=v_{\varepsilon}\left(x, \delta_{\varepsilon}\right)-K u_{\varepsilon}\left(x, \delta_{\varepsilon}\right) .
\end{array}\right.
$$

By construction, $u_{\varepsilon}(x, t)=u_{1, \varepsilon}(x, t)+u_{2, \varepsilon}(x, t)$ and $v_{\varepsilon}(x, t)=v_{1, \varepsilon}(x, t)+$ $v_{2, \varepsilon}(x, t)$ for all $t \geq \delta_{\varepsilon}$. It is easy to show that the solution of (68) tends to zero as $\varepsilon \rightarrow 0$. Indeed, applying the estimate

$$
\|g\|_{L^{2}\left(\partial \Omega_{\varepsilon}\right)}^{2} \leq \frac{c}{\varepsilon}\|g\|_{L^{2}\left(\Omega_{\varepsilon}\right)}^{2}+c \varepsilon\|\nabla g\|_{L^{2}\left(\Omega_{\varepsilon}\right)}^{2}
$$

which is valid for any $g \in H^{1}\left(\Omega_{\varepsilon}\right)$, we conclude that

$$
\left\|K u_{\varepsilon}\left(\cdot, \delta_{\varepsilon}\right)-v_{\varepsilon}\left(\cdot, \delta_{\varepsilon}\right)\right\|_{L^{2}\left(\partial \Omega_{\varepsilon}\right)}^{2} \leq c \varepsilon / \delta_{0} .
$$

Therefore, by the a priori estimate (18) applied to (68), we get

$$
\left\|u_{2, \varepsilon}(\cdot, t)\right\|_{L^{2}\left(\Omega_{\varepsilon}\right)}^{2}+\varepsilon\left\|v_{2, \varepsilon}(\cdot, t)\right\|_{L^{2}\left(\Omega_{\varepsilon}\right)}^{2} \leq c \varepsilon^{2} / \delta_{0}
$$

for all $t \geq \delta_{\varepsilon}$. Under our choice of $\delta_{\varepsilon}$, using the standard extension operator [1], we can assume, without loss of generality, that $u_{\varepsilon}\left(x, \delta_{\varepsilon}\right)$ is defined in the whole $\mathbb{R}^{n}$, satisfies the bound $\left\|u_{\varepsilon}\left(x, \delta_{\varepsilon}\right)\right\|_{H^{1}\left(\mathbb{R}^{n}\right)}^{2} \leq c / \delta_{0}$ and that $u_{\varepsilon}\left(x-(\bar{b} / \varepsilon) \delta_{\varepsilon}, \delta_{\varepsilon}\right)$ converges, as $\varepsilon \rightarrow 0$, to a function $\check{u}^{0}(x)$, such that $\left\|\check{u}^{0}\right\|_{H^{1}\left(\mathbb{R}^{n}\right)}^{2} \leq c / \delta_{0}$.

In exactly the same way as in the case of consistent initial conditions one can show that on the set $\mathbb{R}^{n} \times(\bar{\delta}, T)$ the function $u_{1, \varepsilon}$ strongly two-scale converges with drift $(x, t) \rightarrow(x-(\bar{b} / \varepsilon) t, t)$ towards a solution to the following problem

$$
\begin{gathered}
\left(\left|Y^{0}\right|+K\left|\partial \Sigma^{0}\right|_{n-1}\right) \partial_{t} \check{u}=\operatorname{div}\left(A^{*} \nabla \check{u}\right) \quad \text { in } \mathbb{R}^{n} \times(\bar{\delta}, T), \\
\check{u}(x, \bar{\delta})=\frac{\left|Y^{0}\right|}{\left(\left|Y^{0}\right|+K\left|\partial \Sigma^{0}\right|_{n-1}\right)} \check{u}^{0}(x)+\frac{\left|\partial \Sigma^{0}\right|}{\left(\left|Y^{0}\right|+K\left|\partial \Sigma^{0}\right|_{n-1}\right)} \check{u}^{0}(x) .
\end{gathered}
$$

In particular,

$$
\lim _{\varepsilon \rightarrow 0}\left\|u_{1, \varepsilon}\right\|_{L^{2}\left(\Omega_{\varepsilon} \times\left(\delta_{\varepsilon}, T\right)\right)}^{2}=\left|Y^{0}\right|\|\check{u}\|_{L^{2}\left(\mathbb{R}^{n} \times(\bar{\delta}, T)\right)}^{2} .
$$


Combining this with (69), we deduce that $u_{\varepsilon}$ two-scale converges with drift $(x, t) \rightarrow(x-(\bar{b} / \varepsilon) t, t)$ on the set $\mathbb{R}^{n} \times(\bar{\delta}, T)$ to the function $\check{u}$. This implies that $\check{u}(x, t)=u(x, t)$ for $t \geq \bar{\delta}$, and

$$
\lim _{\varepsilon \rightarrow 0}\left\|u_{\varepsilon}\right\|_{L^{2}\left(\Omega_{\varepsilon} \times\left(t_{\varepsilon}, T\right)\right)}^{2}=\left|Y^{0}\right|\|\check{u}\|_{L^{2}\left(\mathbb{R}^{n} \times(\bar{\delta}, T)\right)}^{2},
$$

then

$$
\lim _{\varepsilon \rightarrow 0}\left\|u_{\varepsilon}\right\|_{L^{2}\left(\Omega_{\varepsilon} \times\left(t_{\varepsilon}, T\right)\right)}^{2}=\left|Y^{0}\right|\|u\|_{L^{2}\left(\mathbb{R}^{n} \times(\bar{\delta}, T)\right)}^{2} .
$$

The last relation contradicts our assumption (66). Thus, we have proved Theorem 3.

\section{References}

[1] E. Acerbi, V. Chiado Piat, G. Dal Maso, D. Percivale, An extension theorem from connected sets, and homogenization in general periodic domains, Nonlinear Anal. 18 (1992), p. 481-496.

[2] G. Allaire, Homogenization and two-scale convergence, SIAM J. Math. Anal. 23, 6, pp.1482-1518 (1992).

[3] G. Allaire, Periodic homogenization and effective mass theorems for the Schrödinger equation, in Quantum transport. Modelling, analysis and asymptotics. N. Ben Abdallah and G. Frosali eds., pp.1-44, Lecture Notes in Mathematics 1946, Springer (2008).

[4] G. Allaire, R. Brizzi, A. Mikelić, A. Piatnitski, Two-scale expansion with drift approach to the Taylor dispersion for reactive transport through porous media, to appear in Chemical Engineering Science, doi:10.1016/j.ces.2009.09.010.

[5] G. Allaire, A. Damlamian and U. Hornung, Two-scale convergence on periodic surfaces and applications, In Mathematical Modelling of Flow through Porous Media, Bourgeat AP, Carasso C, Luckhaus S, Mikelić A (eds). World Scientific: Singapore, 1995; p. 15-25.

[6] G. Allaire, R. Orive, Homogenization of periodic non self-adjoint problems with large drift and potential, COCV 13, pp.735-749 (2007).

[7] G. Allaire, A. Piatnistki, Uniform Spectral Asymptotics for Singularly Perturbed Locally Periodic Operators, Com. in PDE 27, pp.705-725 (2002).

[8] G. Allaire, A.L. Raphael, Homogenization of a convection-diffusion model with reaction in a porous medium. C. R. Math. Acad. Sci. Paris 344 (2007), no. 8, p. 523-528.

[9] J.L. Auriault, P.M. Adler, Taylor dispersion in porous media : Analysis by multiple scale expansions, Advances in Water Resources, Vol. 18 (1995), p. 217-226.

[10] J. Bear, Hydraulics of Groundwater, McGraw-Hill, Jerusalem, 1979. 
[11] A. Bourgeat, M. Jurak, A.L. Piatnitski, Averaging a transport equation with small diffusion and oscillating velocity, Math. Meth. Appl. Sci., Vol. 26 (2003), pp. 95-117.

[12] C. Choquet, A. Mikelić, Laplace transform approach to the rigorous upscaling of the infinite adsorption rate reactive flow under dominant Péclet number through a pore, Applicable Analysis, Vol. 87, No. 12, December 2008, 1373-1395.

[13] C. Conca, On the application of the homogenization theory to a class of problems arising in fluid mechanics, J. Math. Pures Appl. (9) 64 (1985), no. 1, 31-75.

[14] P. Donato, A. Piatnitski, Averaging of nonstationary parabolic operators with large lower order terms, GAKUTO International Series, Math. Sci. Appl., Vol. 24 (2005), Multi Scale Problems and Asymptotic Analysis, pp. 153-165.

[15] C.J. van Duijn, P. Knabner, Travelling Waves in the Transport of Reactive Solutes through Porous Media: Adsorption and Binary Ion Exchange - Part 1, Transport in Porous Media, Vol. 8 (1992), p. 167-194.

[16] C.J. van Duijn, P. Knabner, Travelling wave behavior of crystal dissolution in porous media flow, Euro. Jnl. of Applied Mathematics, Vol. 8 (1997), pp. 49-72.

[17] C.J. van Duijn, P. Knabner, R.J. Schotting, An analysis of crystal dissolution fronts in flows through porous media. Part 2: Incompatible boundary conditions, Adv. Water Resour., Vol. 22 (1998), p. 1-16.

[18] C.J. van Duijn, I.S. Pop, Crystal dissolution and precipitation in porous media : pore scale analysis, J. Reine Angew. Math., Vol. 577 (2004), p. 171-211.

[19] U. Hornung, Homogenization and porous media, Interdisciplinary Applied Mathematics Series, vol. 6, Springer Verlag (1997).

[20] U. Hornung, W. Jäger, Diffusion, convection, adsorption, and reaction of chemicals in porous media, J. Diff. Eq. 92, p. 199-225 (1991).

[21] U. Hornung, W. Jäger, A. Mikelić, Reactive transport through an array of cells with semi-permeable membranes, Math. Mod. Num. Anal. 28 (1), p. 59-94 (1994).

[22] P. Knabner, C.J. van Duijn, S. Hengst, An analysis of crystal dissolution fronts in flows through porous media. Part 1: Compatible boundary conditions, Advances in Water Resources, Vol. 18 (1995), pp. 171-185.

[23] E. Marušić-Paloka, A. Piatnitski, Homogenization of a nonlinear convection-diffusion equation with rapidly oscillating coefficients and strong convection, Journal of London Math. Soc., Vol. 72 (2005), No. 2, p. 391-409.

[24] R. Mauri, Dispersion, convection and reaction in porous media, Phys. Fluids A, Vol. 3 (1991), p. 743-755. 
[25] A. Mikelić, V. Devigne, C.J. van Duijn : Rigorous upscaling of the reactive flow through a pore, under dominant Péclet and Damkohler numbers, SIAM J. Math. Anal., Vol. 38, Issue 4 (2006), p. 1262-1287.

[26] G. Nguetseng, A general convergence result for a functional related to the theory of homogenization, SIAM J. Math. Anal. 20(3), 608-623 (1989).

[27] M. Neuss-Radu, Some extensions of two-scale convergence. C. R. Acad. Sci. Paris Sr. I Math. 322 (1996), no. 9, p. 899-904.

[28] G. C. Papanicolaou, Diffusion in random media, Surveys in applied mathematics, Vol. 1, 205-253, Plenum, New York, 1995.

[29] M.A. Paine, R.G. Carbonell, S. Whitaker, Dispersion in pulsed systems - I, Heterogeneous reaction and reversible adsorption in capillary tubes, Chemical Engineering Science, Vol. 38 (1983), p. 1781-1793.

[30] M. Panfilov, Macroscale Models of Flow Through Highly Heterogeneous Porous Media, Kluwer Academic (2000).

[31] L. Pankratov, A. Piatnitskii, V. Rybalko, Homogenized model of reactiondiffusion in a porous medium, C. R. Mécanique 331, p. 253-258 (2003).

[32] M. Peszyńska, R.E. Showalter, Macroscale Models of Flow Through Highly Heterogeneous Porous Media, EJDE 147, p. 1-30 (2007).

[33] M.A. Peter, M. Böhm, Different choices of scaling in homogenization of diffusion and interfacial exchange in a porous medium, Math. Meth. Appl. Sci. 31, p. 1257-1282 (2008).

[34] A.L. Piatnitski, Averaging singularly perturbed equation with rapidly oscillating coefficients in a layer, Math. USSR Sbornik, Vol. 49 (1984), p. $19-40$.

[35] J. Rubinstein, R. Mauri, Dispersion and convection in porous media, SIAM J. Appl. Math. , Vol. 46 (1986), p. 1018 - 1023.

[36] G.I. Taylor, Dispersion of soluble matter in solvent flowing slowly through a tube, Proc. Royal Soc. A, Vol. 219 (1953), p. 186-203. 